\title{
Helly Property, Clique Graphs, Complementary Graph Classes, and Sandwich Problems*
}

\author{
Mitre C. Dourado ${ }^{1}$ Priscila Petito $^{2}$, Rafael B. Teixeira ${ }^{2}$ and Celina M. H. de Figueiredo ${ }^{2}$ \\ ${ }^{1}$ ICE, Universidade Federal Rural do Rio de Janeiro e NCE, Universidade Federal do Rio de Janeiro \\ BR-465, Km 7, 23890-000, Seropedica, RJ, Brasil
}

\{mitre\}@nce.ufrj.br

${ }^{2}$ Programa de Engenharia de Sistemas e Computação, COPPE, Universidade Federal do Rio de Janeiro Caixa Postal 68511, 21945-970, Rio de Janeiro, RJ, Brasil

\{ppetito I rafaelbt I celina \}@ cos.ufrj.br

Received 18 January 2008; accepted 12 May 2008

\begin{abstract}
A sandwich problem for property $\Pi$ asks whether there exists a sandwich graph of a given pair of graphs which has the desired property П. Graph sandwich problems were first defined in the context of Computational Biology as natural generalizations of recognition problems. We contribute to the study of the complexity of graph sandwich problems by considering the Helly property and complementary graph classes. We obtain a graph class defined by a finite family of minimal forbidden subgraphs for which the sandwich problem is NP-complete. A graph is clique-Helly when its family of cliques satisfies the Helly property. A graph is hereditary clique-Helly when all of its induced subgraphs are clique-Helly. The clique graph of a graph is the intersection graph of the family of its cliques. The recognition problem for the class of clique graphs was a long-standing open problem that was recently solved. We show that the sandwich problems for the graph classes: clique, clique-Helly, hereditary clique-Helly, and clique-Helly nonhereditary are all $N P$-complete. We propose the study of the complexity of sandwich problems for complementary graph classes as a mean to further understand the sandwich problem as a generalization of the recognition problem.
\end{abstract}

Keywords: Helly property, Clique graphs, Sandwich problems, Computational difficulty of problems.

${ }^{*}$ An extended abstract was presented at 7 th International Colloquium on Graph Theory, Electron. Notes Discrete Math. 22 (2005) 497-500.

\section{INTRODUCTION}

We consider simple, finite, undirected graphs. Given a graph $G=(V, E)$, a complete set of $G$ is a subset of $V$ inducing a complete subgraph. A clique is a maximal complete set. Let $\mathcal{F}$ be a family of subsets of a set $S$. We say that $\mathcal{F}$ satisfies the Helly property when every subfamily consisting of pairwise intersecting subsets has a non-empty intersection. The Helly property has an important role in Combinatorics and its computational aspects [5]. A graph is clique-Helly when its family of cliques satisfies the Helly property. A graph is hereditary clique-Helly when all of its induced subgraphs are clique-Helly. This graph class admits a finite family of minimal forbidden subgraphs. A clique-Helly graph that is not hereditary clique-Helly is called clique-Helly nonhereditary. The clique graph of a graph is the intersection graph of the family of its cliques. $G$ is a clique graph if there exists a graph $H$ such that $G$ is the clique graph of $H$. The recognition problems for the classes of cliqueHelly graphs and of hereditary clique-Helly graphs are known to be polynomial. The recognition problem for the class of clique graphs was a long-standing open problem $[3,14,16,18]$ that was recently solved [1]. Clique graphs have been much studied as intersection graphs, and in the context of graph operators, and are included in several books $[3,12,15]$.

We consider the following generalization of recognition problems. We say that a graph $G_{1}=\left(V, E_{1}\right)$ is a spanning subgraph of $G_{2}=\left(V, E_{2}\right)$ if $E_{1} \subseteq E_{2}$; and that 
a graph $G=(V, E)$ is a sandwich graph for the pair $G_{1}$, $G_{2}$ if $E_{1} \subseteq E \subseteq E_{2}$. We call $E_{1}$ the forced edge set, $E_{2} \backslash E_{1}$ the optional edge set. The GRAPH SANDWICH PROBLEM FOR PROPERTY $\Pi$ is defined as follows [8]:

GRAPH SANDWICH PROBLEM FOR PROPERTY П

Instance: Vertex set $V$, forced edge set $E_{1}$, optional edge set $E_{2} \backslash E_{1}$.

Question: Is there a graph $G=(V, E)$ such that $E_{1} \subseteq E \subseteq E_{2}$ that satisfies property $\Pi$ ?

Graph sandwich problems were defined in the context of Computational Biology and have attracted much attention lately arising from many applications and as a natural generalization of recognition problems [4, 7, 8, 9, 11]. The recognition problem for a class of graphs $\mathcal{C}$ is equivalent to the graph sandwich problem in which the forced edge set $E_{1}=E$, the optional edge set $E_{2} \backslash E_{1}=\emptyset$, $G=(V, E)$ is the graph we want to recognize, and property $\Pi$ is "to belong to class $\mathcal{C}$ ".

Golumbic et al. [8] have considered sandwich problems with respect to several subclasses of perfect graphs, and proved that the GRAPH SANDWICH PROBLEM FOR SPLIT GRAPHS remains in $P$. On the other hand, they proved that the GRAPH SANDWICH PROBLEM FOR PERMUTATION GRAPHS turns out to be $N P$-complete.

Clique graphs admit a characterization by a special edge cover. A complete set $C$ covers the edge $u v$ if the endvertices $u$ and $v$ belong to $C$. A complete edge cover of $G$ is a family of complete sets covering all edges of $G$.

Theorem 1 (Roberts and Spencer [16]) A graph $G$ is a clique graph if and only if there exists a complete edge cover of $G$ satisfying the Helly property.

Such a family of complete sets is called an $R S$-family for $G=(V, E)$. If $G$ admits an $R S$-family, then $G$ admits a small $R S$-family with at most $|E|$ sets, because every edge needs to be covered by only one complete set and any subfamily of a family satisfying the Helly property does satisfy the Helly property too. Therefore, the characterization given in Theorem 1 gives a short certificate for the class of clique graphs because the Helly property for a family of subsets can be tested in polynomial time on the size of the family $[2,16,18]$. On the other hand, recognizing clique graphs was recently shown to be $N P$ complete [1].

The family of all cliques of $G$ is a complete edge cover of $G$, so it follows that clique-Helly graphs are clique graphs. A polynomial-time recognition algorithm for the class of clique-Helly graphs was presented in [6] and independently in [17]. This algorithm is a consequence of a characterization of clique-Helly graphs based on the concept of extended triangle. A triangle $T$ of a graph $G$ is a complete set containing exactly three vertices. The $e x$ tended triangle of $G$ relative to the triangle $T$ is defined in [17] as the subgraph induced in $G$ by the vertices adjacent to at least two vertices of $T$ and it is denoted $T^{\prime}$. A universal vertex in a graph is adjacent to every other vertex of the graph.

Theorem 2 (Dragan [6], Szwarcfiter [17]) A graph G is a clique-Helly graph if and only if every extended triangle of $G$ contains a universal vertex.

A polynomial-time recognition algorithm for the class of hereditary clique-Helly graphs was presented in [13]. This algorithm uses a finite family of induced forbidden subgraphs, the so-called ocular graphs depicted in Figure 1.

Given a graph $G$ and a triangle $T=\left\{t_{1}, t_{2}, t_{3}\right\}$, we say that $T$ yields an ocular graph in $G$ if there are vertices $s_{1}, s_{2}$ and $s_{3}$ such that vertex $s_{1}$ is not adjacent to $t_{1}$ and is adjacent to $t_{2}$ and $t_{3}$, vertex $s_{2}$ is not adjacent to $t_{2}$ and is adjacent to $t_{1}$ and $t_{3}$, and vertex $s_{3}$ is not adjacent to $t_{3}$ and is adjacent to $t_{1}$ and $t_{2}$. The graph on the left of Figure 1 is known as the Hajós graph and the triangle that yields this particular ocular graph is known as the central triangle of this Hajós graph. Ocular graphs were defined in [19] as a tool to study the Helly property and were shown in [13] to be the minimal forbidden configurations for hereditary clique-Helly graphs.

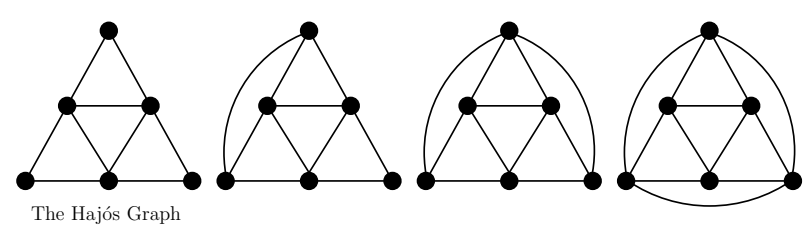

Figure 1. The ocular graphs are the minimal forbidden induced subgraphs for a hereditary clique-Helly graph

Theorem 3 (Prisner [13]) A graph $G$ is a hereditary clique-Helly graph if and only if $G$ contains none of the four graphs shown in Figure 1 as an induced subgraph.

Clearly, from Theorems 2 and 3 one can also recognize in polynomial time whether a graph is clique-Helly nonhereditary.

We contribute to the study of the complexity of graph sandwich problems by considering the Helly property and complementary graph classes. Surprisingly, we obtain a graph class defined by a finite family of minimal forbidden subgraphs for which the corresponding sandwich problem is $N P$-complete. Additionally, we propose the study of the complexity of sandwich problems for complementary graph classes as a mean to further understand 
the sandwich problem as a generalization of the recognition problem.

The studies on sandwich problems focus on properties that are neither hereditary nor ancestral on subgraphs, in which case the complexity of the recognition problem and of the corresponding sandwich problem coincide. The graph classes considered in the present paper: clique, clique-Helly, hereditary clique-Helly are neither hereditary nor ancestral on subgraphs. Most properties considered so far are hereditary on induced subgraphs, which means that the corresponding graph class admits a family of minimal forbidden subgraphs. In particular, every studied sandwich problem corresponding to a graph class defined by a finite family of minimal forbidden subgraphs has been classified as Polynomial. Note the classes clique and clique-Helly are not hereditary on induced subgraphs. The class hereditary clique-Helly is the first example of a graph class defined by a finite family of minimal forbidden subgraphs for which the corresponding sandwich problem is $N P$-complete.

This paper is organized as follows: in Section 2 we prove that HEREDITARY CLIQUE-HELLY SANDWICH PROBLEM, CLIQUE-HELLY SANDWICH PROBLEM, and CLIQUE GRAPH SANDWICH PROBLEM are $N P$-complete; in Section 3 we prove that CLIQUEHELLY NONHEREDITARY SANDWICH PROBLEM is $N P$ complete; in Section 4 we have our concluding remarks about complementary graph classes and sandwich problems.

\section{Clique, Clique-Helly, and HEREDITARY CLIQUE-HELLY SANDWICH PROBLEMS}

We prove that the HEREDITARY CLIQUE-HELLY SANDWICH PROBLEM is $N P$-complete by a reduction from the $N P$-complete problem 3-SAT. These two decision problems are defined as follows.

3-SAT

Instance: Set $X=\left\{x_{1}, \ldots, x_{n}\right\}$ of variables, collection $C=\left\{c_{1}, \ldots, c_{m}\right\}$ of clauses over $X$ such that each clause $c \in C$ has $|c|=3$ literals.

Question: Is there a truth assignment for $X$ such that each clause in $C$ has at least one true literal?

HEREDITARY CLIQUE-HELLY SANDWICH PROBLEM

Instance: Vertex set $V$, forced edge set $E_{1}$, optional edge set $E_{2} \backslash E_{1}$.

Question: Is there a graph $G=(V, E)$, such that $E_{1} \subseteq E \subseteq E_{2}$ and $G$ is hereditary

\author{
clique-Helly?
}

Theorem 4 The HEREDITARY CLIQUE-HELLY SANDWICH PROBLEM is NP-complete.

Proof. The HeREditary CLIQUe-HELly SANDWICH PROBLEM is in $N P$ since a short certificate is a sandwich graph $G$ that is hereditary clique-Helly together with the polynomial-time algorithm for hereditary cliqueHelly recognition.

In order to reduce 3-SAT to HEREDITARY CLIQUEHELLY SANDWICH PROBLEM we need to construct in polynomial time a particular instance $\left(V, E_{1}, E_{2}\right)$ of HEREDITARY CLIQUE-HELLY SANDWICH PROBLEM from a generic instance $(X, C)$ of 3 -SAT, such that $C$ is satisfiable if and only if $\left(V, E_{1}, E_{2}\right)$ admits a sandwich graph $G=(V, E)$ which is hereditary clique-Helly. Recall the notation $G_{1}=\left(V, E_{1}\right)$ and $G_{2}=\left(V, E_{2}\right)$.

Let $(X, C)$ be a generic instance of 3 -SAT. We assume without loss of generality that each variable occurs both as a positive and as a negative literal. For each variable $x_{i}$, add to $V$ four variable vertices: $X_{i}, \bar{X}_{i}, a_{i}$ and $b_{i}$. For each clause $c_{j}=\left\{\ell_{r}, \ell_{s}, \ell_{t}\right\}$, where $\ell_{u}, u \in\{r, s, t\}$, is a literal of variable $x_{u}$, add to $V$ clause vertices $y_{j r}$, $y_{j s}, y_{j t}$. The set $V$ contains $4 n$ variable vertices, plus $3 m$ clause vertices.

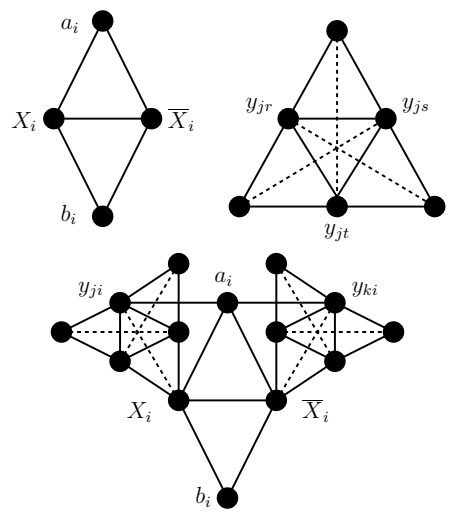

Figure 2. Forced variable gadget; forced Hajós graph corresponding to clause $c_{j}$; and Hajós graph in $G_{2}$, induced by vertices $X_{i}, \bar{X}_{i}, a_{i}, b_{i}$, $y_{j i}$ and $y_{k i}$, corresponding to variable $x_{i}$

Please refer to Figure 2, where we have depicted the forced edges as continuous edges, and the optional edges as dotted edges. Each triple of clause vertices corresponding to a clause $c_{j}$ induce a triangle $T_{j}$ in $G_{1}$, called clause triangle. Vertices $a_{i}, b_{i}, X_{i}, \bar{X}_{i}$ induce a forced variable gadget $D_{i}$ in $G_{1}$, composed by two triangles that share one edge $X_{i} \bar{X}_{i}$.

Let $y_{j r}, y_{j s}, y_{j t}$ be the clause vertices of a clause triangle $T_{j}$. If the clause vertex $y_{j u}, u \in\{r, s, t\}$, corresponds to the positive literal of variable $x_{u}$, then add optional 
edge $X_{u} y_{j u}$, add two forced edges $X_{u} y_{j w}$, for $w \neq u$, and add forced edge $a_{u} y_{j u}$. If the clause vertex $y_{j u}$, $u \in\{r, s, t\}$ corresponds to the negative literal of variable $x_{u}$, then add optional edge $\bar{X}_{u} y_{j u}$, add two forced edges $\bar{X}_{u} y_{j w}$, for $w \neq u$, and add forced edge $a_{u} y_{j u}$. The construction of the particular instance $\left(V, E_{1}, E_{2}\right)$ is concluded.

First, we show that, given a sandwich graph $G^{\prime}$ of $G_{1}$ and $G_{2}$, if $G^{\prime}$ contains an ocular graph as induced subgraph, then this ocular graph is in fact a Hajós graph. Recall that a triangle $T=\left\{t_{1}, t_{2}, t_{3}\right\}$ yields an ocular graph if there are vertices $s_{1}, s_{2}$ and $s_{3}$ such that vertex $s_{1}$ is not adjacent to $t_{1}$ and is adjacent to $t_{2}$ and $t_{3}$, vertex $s_{2}$ is not adjacent to $t_{2}$ and is adjacent to $t_{1}$ and $t_{3}$, and vertex $s_{3}$ is not adjacent to $t_{3}$ and is adjacent to $t_{1}$ and $t_{2}$. Furthermore, we show there are only two cases for a triangle to yield an ocular graph.

Please refer to Figure 2. First, note that if the triangle $T=\left\{y_{j r}, y_{j s}, y_{j t}\right\}$, formed by the three clause vertices of a clause $c_{j}$, yields an ocular graph in $G^{\prime}$, this subgraph is in fact a Hajós graph, because there are no edges joining two variable vertices of distinct variables. We call such a subgraph of clause Hajós graph.

Now, see that if the triangle $T=\left\{a_{i}, X_{i}, \bar{X}_{i}\right\}$, formed by three variable vertices of a variable $x_{i}$, yields an ocular graph in $G^{\prime}$, this subgraph is also a Hajós graph, because $b_{i}$ has degree two in $G_{2}$ and there are no edges joining two clause vertices of distinct clauses. We call such a graph of variable Hajós graph.

The only other two possibilities of a triangle $T$ are: $T=\left\{b_{i}, X_{i}, \bar{X}_{i}\right\}$, for some $i$; and $T=\{v, l, z\}$, where $v$ is a variable vertex, $l$ is a clause vertex, and $z$ is a variable or a clause vertex. In the first possibility $T=\left\{b_{i}, X_{i}, \bar{X}_{i}\right\}$, for some $i$, since $b_{i}$ has degree two in $G_{2}$, such $T$ cannot yield an ocular graph in $G^{\prime}$. In the second possibility $T=\{v, l, z\}$, where $v$ is a variable vertex, $l$ is a clause vertex, and $z$ is a variable or a clause vertex, since $a_{i}$ and $y_{j i}$ have just one common neighbor in $G_{2}$ and since the only common neighbors of $y_{j r}$ and $X_{s}$ in $G_{2}$ are $y_{j s}$ and $y_{j t}$ it is clear that such $T$ cannot yield an ocular graph in $G^{\prime}$.

We shall use the characterization of Theorem 3. By the construction, it is clear that $G_{1}$ and $G_{2}$ contain, respectively, a clause Hajós graph and a variable Hajós graph, hence $G_{1}$ and $G_{2}$ are not hereditary clique-Helly.

Suppose first that $C$ is satisfiable, and consider a truth assignment for $X$ such that each clause $c_{j}$ in $C$ has at least one true literal. To define the sandwich graph $G$, if $x_{i}$ has value true, add to $E$, for every clause $c_{j}$ where $x_{i}$ occurs as positive literal, the optional edge $y_{j i} X_{i}$; if $x_{i}$ has value false, add to $E$, for every clause $c_{j}$ where $x_{i}$ occurs as negative literal, the optional edge $y_{j i} \bar{X}_{i}$. Note that in the constructed sandwich graph $G$, for each variable $x_{i}$, either all optional edges incident to vertex $X_{i}$ or all optional edges incident to vertex $\bar{X}_{i}$ are present. Therefore, each variable Hajós graph in $G_{2}$ is avoided in $G$. In addition, note that in the constructed sandwich graph $G$, for each clause $c_{j}$, at least one optional edge is added to the corresponding forced clause Hajós graph. Therefore, each clause Hajós graph in $G_{1}$ is avoided in $G$.

Conversely, suppose that $G$ is a hereditary cliqueHelly sandwich graph for the particular constructed instance $\left(V, E_{1}, E_{2}\right)$. It follows that $G$ contains none of the forbidden configurations depicted in Figure 1. By the construction of the particular instance $\left(V, E_{1}, E_{2}\right)$, graph $G_{1}$ contains a forced clause Hajós graph corresponding to each clause. Therefore, in the hereditary clique-Helly sandwich graph $G$, for each forced clause Hajós graph, we must have at least one added optional edge. In addition, $G$ cannot have for a variable $x_{i}$, both an optional edge incident to vertex $X_{i}$ and an optional edge incident to vertex $\bar{X}_{i}$ present, as this would give a variable Hajós graph. Therefore we can construct a truth assignment by setting variable $x_{i}$ as true if there exists in $G$ an edge $y_{j i} X_{i}$ for some clause $c_{j}$ where $x_{i}$ occurs as positive literal, and by setting variable $x_{i}$ as false otherwise.

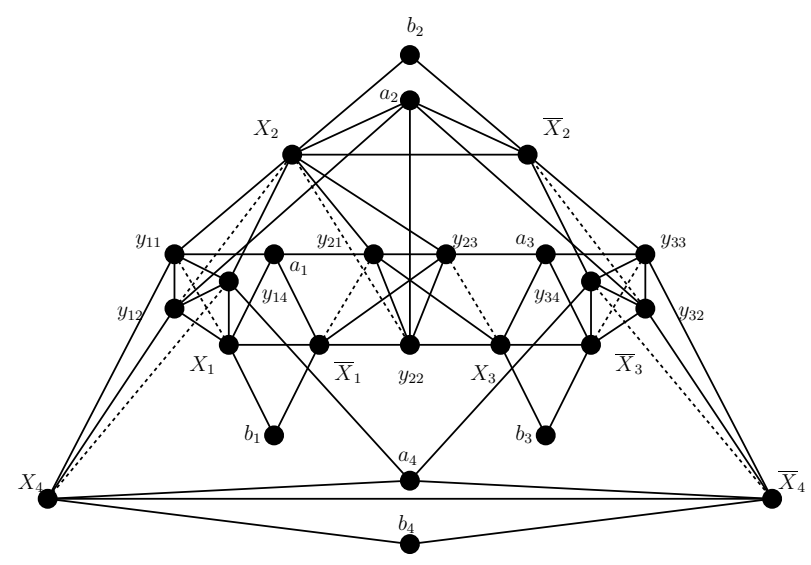

Figure 3. Example of constructed instance of $\left(V, E_{1}, E_{2}\right)$ corresponding to clauses $\left\{x_{1}, x_{2}, x_{4}\right\},\left\{\bar{x}_{1}, x_{2}, x_{3}\right\},\left\{\bar{x}_{2}, \bar{x}_{3}, \bar{x}_{4}\right\}$

Figure 3 shows an example of an instance constructed in Theorem 4. We have depicted the forced edges as continuous edges, and the optional edges as dotted edges.

In the sequel, we further study the properties of the particular instance constructed in Theorem 4 and prove in Theorem 5 and Theorem 6, respectively, that the CLIQUEHELLY SANDWICH PROBLEM and CLIQUE SANDWICH PROBLEM are $N P$-complete.

\section{CLIQUE-HELLY SANDWICH PROBLEM}

Instance: Vertex set $V$, forced edge set $E_{1}$, optional edge set $E_{2} \backslash E_{1}$. 
Question: Is there a graph $G=(V, E)$, such that $E_{1} \subseteq E \subseteq E_{2}$ and $G$ is clique-Helly?

Theorem 5 The CLIQUE-HELLY SANDWICH PROBLEM is NP-complete.

Proof. The Clique-Helly SANDWich PROBlem is in $N P$ since a short certificate is a sandwich graph $G$ that is clique-Helly together with the polynomial-time algorithm for clique-Helly recognition.

In order to establish the hardness of the problem, we perform the same reduction from 3-SAT of Theorem 4. It is sufficient to show that every sandwich graph of $\left(V, E_{1}, E_{2}\right)$ which is clique-Helly is also hereditary clique-Helly. By contradiction, suppose that a sandwich graph $G$ of $\left(V, E_{1}, E_{2}\right)$ is clique-Helly but is not hereditary clique-Helly. By Theorem 3, $G$ has as induced subgraph one of the graphs of Figure 1. Denote it by $G^{\prime}$. By the observations present in the proof of Theorem 4, $G^{\prime}$ is indeed either a clause Hajós graph or a variable Hajós graph. In both cases there are two variable vertices $v_{1}$ and $v_{2}$ in $G^{\prime}$. Note these two variable vertices can be of a same variable or not. Since $G$ is clique-Helly, by Theorem 2 , there must exist a vertex $v$ adjacent to every vertex of $G^{\prime}$. Recall every vertex of $V$ is a clause vertex or a variable vertex. Since the degree of a clause vertex is at most 6 , and in this case, its 6 neighbors do not induce a Hajós graph, we conclude that $v$ is a variable vertex. We have a contradiction, because there is no variable vertex outside of $G^{\prime}$ adjacent to $v_{1}$ and $v_{2}$.

Now, we turn our attention to the CLIQUE GRAPH SANDWICH PROBLEM and we present a simple proof of its $N P$-completeness.

\section{CLIQUE GRAPH SANDWICH PROBLEM \\ Instance: Vertex set $V$, forced edge set $E_{1}$, op- tional edge set $E_{2} \backslash E_{1}$. \\ Question: Is there a graph $G=(V, E)$, such that $E_{1} \subseteq E \subseteq E_{2}$ and $G$ is clique graph?}

First we need an auxiliary lemma. This lemma is a consequence of a more general result established in [10].

Lemma 1 Let $G$ be a graph containing a Hajós Graph $H$ as an induced subgraph. If the central triangle $T$ of $H$ is not contained in a $K_{4}$, then $G$ is not a clique graph.

Proof. Assume to get a contradiction that we have an $R S$-family $\mathcal{C}$ for $G$. We consider two cases with respect to the central triangle $T$ of $H$ to belong to $\mathcal{C}$ or not. Let $h_{1}, h_{2}, h_{3}$ be the vertices of $T$, and $h_{4}, h_{5}, h_{6}$ the other vertices of $H$, such that $h_{4} h_{2}, h_{4} h_{3}, h_{5} h_{1}, h_{5} h_{2}, h_{6} h_{1}, h_{6} h_{3} \in E$. In case vertices $h_{i}, h_{j}, h_{k}$ define a triangle, denote this triangle by $T_{i j k}$. Let $H_{p q}$ be a complete set of $\mathcal{C}$ covering edge $h_{p} h_{q}$.
First, suppose that $T \notin \mathcal{C}$. Since $T$ is not contained in a $K_{4}$, the subfamily of distinct complete sets $H_{12}, H_{13}, H_{23}$ of $\mathcal{C}$ does not satisfy the Helly property, a contradiction.

Next, suppose that $T \in \mathcal{C}$. If $h_{2} \notin H_{34}$ and $h_{3} \notin H_{24}$, then $\mathrm{H}_{24}, \mathrm{H}_{34}$ and $\mathrm{T}$ do not satisfy the Helly property. Therefore, there are complete sets $C_{1}, C_{2}, C_{3} \in \mathcal{C}$ such that $h_{1}, h_{3}, h_{6} \in C_{1}, h_{1}, h_{2}, h_{5} \in C_{2}$, and $h_{2}, h_{3}, h_{4} \in$ $C_{3}$. And $C_{1}, C_{2}, C_{3}$ contain a common vertex if and only if $T \subset K_{4}$, a contradiction.

Theorem 6 The CLIQUE GRAPH SANDWICH PROBLEM is NP-complete.

Proof. The CLIQUe GRAPH SANDWICH PROBLEM is in $N P$ since a short certificate is a sandwich graph $G$ that is a clique graph, and a small $R S$-family for $G$ together with the polynomial-time algorithm for the Helly property.

Again we perform a reduction from 3-SAT by using the same particular instance of Theorem 4. Let $\left(V, E_{1}, E_{2}\right)$ be the particular instance constructed. Since, by Theorem 1 , every hereditary clique-Helly graph is a clique graph, it is sufficient to show that every sandwich graph of $\left(V, E_{1}, E_{2}\right)$ which is a clique graph is also hereditary clique-Helly. By contradiction, suppose that the sandwich graph $G$ of $\left(V, E_{1}, E_{2}\right)$ is a clique graph but is not hereditary clique-Helly. Hence, by Theorem 3 and the observations of Theorem 4, $G$ contains either a clause Hajós graph or a variable Hajós graph as induced subgraph. Denote it by $G^{\prime}$. Since $G$ is a clique graph, by Lemma 1 , the central triangle of $G^{\prime}$ must be contained in a $K_{4}$. If $G^{\prime}$ is a clause Hajós graph, then its central triangle is of the form $T=\left\{y_{k r}, y_{k s}, y_{k t}\right\}$. Since, given $u \in\{r, s, t\}$, the only vertex adjacent to $y_{k u}$ and not contained in $G^{\prime}$ is a variable vertex $a_{u}$, and $a_{u}$ is nonadjacent to the other vertices of $T$, this central triangle is not in a $K_{4}$. Finally, if $G^{\prime}$ is a variable Hajós graph, its central triangle is of the form $\left\{a_{i}, X_{i}, \bar{X}_{i}\right\}$. This central triangle is not in a $K_{4}$ because there is no vertex adjacent in $G_{2}$ to these three vertices.

\section{Clique-Helly SANDWICH PROBLEM \\ NONHEREDITARY}

We cannot conclude from Theorems 4 and 5 that the sandwich problem for clique-Helly nonhereditary graphs is NP-complete. Theorem 7 proves that the CLIQUEHELLY NONHEREDITARY SANDWICH PROBLEM is $N P$ complete by a reduction from the $N P$-complete problem SAT. These two decision problems are defined as follows. 


\section{SAT}

Instance: Set $X=\left\{x_{1}, \ldots, x_{n}\right\}$ of variables, collection $C=\left\{c_{1}, \ldots, c_{m}\right\}$ of clauses over $X$.

Question: Is there a truth assignment for $X$ such that each clause in $C$ has at least one true literal?

\section{CLIQUE-HELLY NONHEREDITARY SAND- WICH PROBLEM \\ Instance: Vertex set $V$, forced edge set $E_{1}$, op- tional edge set $E_{2} \backslash E_{1}$. \\ Question: Is there a graph $G=(V, E)$, such that $E_{1} \subseteq E \subseteq E_{2}$ and $G$ is clique-Helly nonhereditary?}

Theorem 7 The CLIQUE-HELLY NONHEREDITARY SANDWICH PROBLEM is NP-complete.

Proof. The CliQue-Helly nONHEREDITARY SANDWICH PROBLEM is in $N P$ since a short certificate is a sandwich graph $G$ that is clique-Helly nonhereditary together with the polynomial-time algorithms for cliqueHelly and hereditary clique-Helly recognition.

Let $(X, C)$ be a generic instance of SAT. We assume without loss of generality that each variable occurs both as a positive and as a negative literal.

Each clause $c_{j}$ corresponds to a copy $H_{j}$ of the Hajós graph in $G_{1}$. Each pair of distinct clauses $c_{j}, c_{k}$ correspond to an auxiliary vertex $d_{j k}$ adjacent in $G_{1}$ to two vertices in each of the central triangles of the corresponding Hajós graphs $H_{j}$ and $H_{k}$ (see Figure 4).

As each variable $x_{i}$ occurs both as a positive and as a negative literal, for each variable $x_{i}$, we have three vertices: literal vertex $x_{i}$ represents the occurrences of variable $x_{i}$ as a positive literal, literal vertex $\bar{x}_{i}$ represents the occurrences of variable $x_{i}$ as a negative literal, and vertex $X_{i}$ represents the variable. These three vertices $x_{i}, \bar{x}_{i}, X_{i}$ induce a variable triangle $T_{i}$ in $G_{1}$.

We conclude the construction of particular instance $\left(V, E_{1}, E_{2}\right)$ by defining the optional edge set $E_{2} \backslash E_{1}$. Suppose literal $\ell \in c_{j}$. It corresponds to literal vertex $\ell \in V$. We add to $E_{2} \backslash E_{1}$ six optional edges $\ell z$, one for each vertex $z$ of the Hajós graph $H_{j}$. In addition, for each auxiliary vertex $d_{j k}$, add to $E_{2} \backslash E_{1}$ the optional edge $\ell d_{j k}$.

Note that every sandwich graph of $\left(V, E_{1}, E_{2}\right)$ is not hereditary clique-Helly, because for each clause there is a Hajós graph as induced subgraph of it. Therefore, any sandwich graph of $\left(V, E_{1}, E_{2}\right)$ is clique-Helly nonhereditary if and only if it is clique-Helly. We shall use the characterization of Theorem 2 for clique-Helly graphs.

Suppose first that $C$ is satisfiable, and consider a truth assignment for $X$ such that each clause $c_{j}$ in $C$ has at least one true literal. To define the sandwich graph $G$, add to $E$, for each Hajós graph $H_{j}$ corresponding to clause $c_{j}$, the optional edges that make precisely one of the true literals of clause $c_{j}$ adjacent to every vertex of $H_{j}$, and adjacent to every vertex $d_{j k}$. Let $L$ be the set of the chosen true literals. Note that every added optional edge has one endpoint in $L$. And for an extended triangle present in $G_{1}$, say $T^{\prime}$, with a vertex $z$, if $\ell z$, with $\ell \in L$, was added to $E$ in the construction of $G$, then $\ell z^{\prime}$, for every vertex $z^{\prime} \in V\left(T^{\prime}\right)$, was also added to $E$. This implies that all the extended triangles of $G_{1}$ have universal vertices in $G$. It remains to analyze the extended triangles relative to triangles of $G$ not present in $G_{1}$. Every such triangle contains precisely one literal vertex and this vertex is universal with respect to the relative extended triangle.

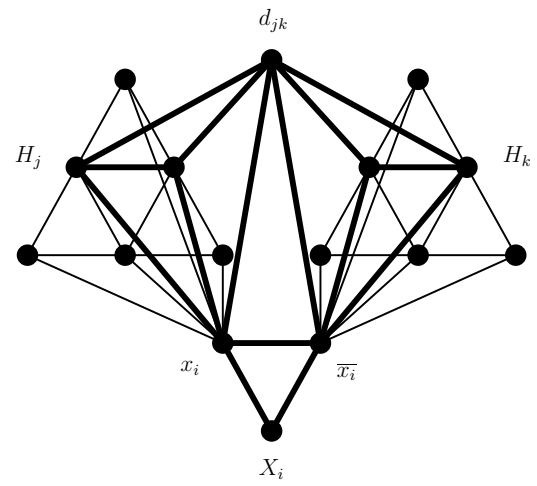

Figure 4. In $G_{2}$ extended triangle relative to triangle $\left\{x_{i}, \bar{x}_{i}, d_{j k}\right\}$ has no universal vertex

Conversely, suppose that $G$ is a clique-Helly sandwich graph for the particular constructed instance $\left(V, E_{1}, E_{2}\right)$. Thus, every extended triangle of $G$ has a universal vertex. Let $c_{j}$ be a clause, and consider its corresponding Hajós graph $H_{j}$. In $G$ the extended triangle relative to the central triangle of $H_{j}$ has a universal vertex. This universal vertex is a literal vertex corresponding to a literal of clause $c_{j}$. For a variable $x_{i}$, suppose that its positive literal occurs in $c_{j}$ and its negative literal occurs in $c_{k}$. If in $G$ both literal vertices $x_{i}$ and $\bar{x}_{i}$ are universal with respect to corresponding Hajós graphs $H_{j}$ and $H_{k}$, then the extended triangle relative to $x_{i}, \bar{x}_{i}, d_{j k}$ has no universal vertex. See Figure 4. Therefore, for each variable $x_{i}$, if its positive literal vertex is universal with respect to a Hajós graph associated to a clause, then set variable $x_{i}$ as true, otherwise set the variable $x_{i}$ as false. We have the desired truth assignment.

Figure 5 shows an example of an instance constructed in the proof of Theorem 7. We have depicted the forced edges as continuous edges, and the optional edges incident to $d_{j k}$ as dotted edges. Each Hajós graph $H_{j}$ is drawn inside a square and we have depicted the six optional edges that make literal $\ell \in c_{j}$ universal with re- 


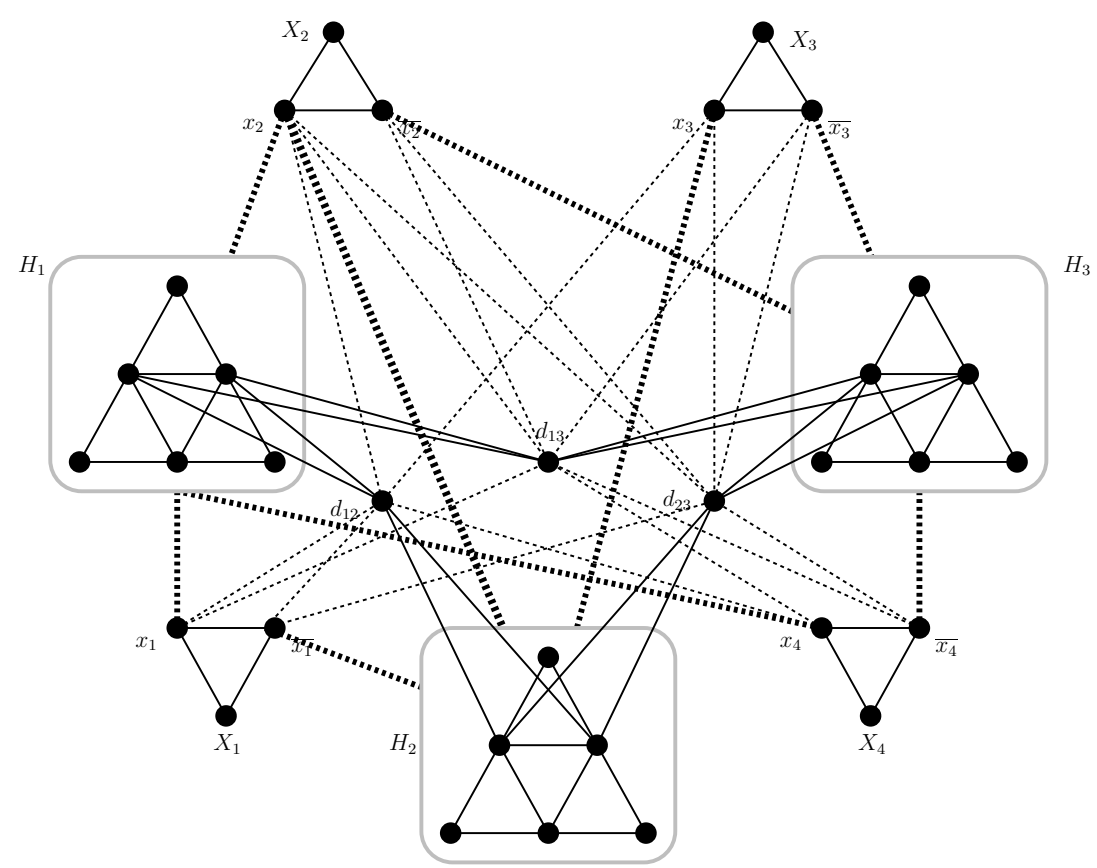

Figure 5. Example of constructed instance of $\left(V, E_{1}, E_{2}\right)$ corresponding to clauses $\left\{x_{1}, x_{2}, x_{4}\right\},\left\{\bar{x}_{1}, x_{2}, x_{3}\right\},\left\{\bar{x}_{2}, \bar{x}_{3}, \bar{x}_{4}\right\}$

spect to the Hajós graph $H_{j}$ by a dotted line joining $\ell$ to the square.

\section{Concluding Remarks}

We have presented four $N P$-completeness proofs, establishing that CLIQUE-HELLY SANDWICH PROBLEM, HEREDITARY CLIQUE-HELLY SANDWICH PROBLEM, CLIQUE-HELLY NONHEREDITARY SANDWICH PROBLEM, and CLIQUE GRAPH SANDWICH PROBLEM are $N P$-complete. We emphasize that the $N P$-completeness of clique graph recognition is a recently solved problem and directly implies the $N P$-completeness of CLIQUE GRAPH SANDWICH PROBLEM. The present paper proposed a simple $N P$-completeness proof for the sandwich version of the problem.

One interesting line of research is to investigate other complementary graph classes with respect to the complexity of the corresponding sandwich problems. It is well-known that one can decide in polynomial time if a graph is chordal and if it is interval. Therefore one can also decide in polynomial time if such a graph is chordal but not interval. The complexity of the corresponding sandwich problems: GRAPH SANDWICH PROBLEM FOR CHORDAL GRAPHS and GRAPH SANDWICH PROBLEM FOR INTERVAL GRAPHS was considered in [8], where both sandwich problems are proved to be $N P$-complete. In this case we can conclude that the GRAPH SANDWICH PROBLEM FOR CHORDAL NONINTERVAL GRAPHS is also $N P$-complete. More generally, consider three graph classes, say $\mathcal{C}_{1}, \mathcal{C}_{2}, \mathcal{C}_{3}$, such that $\mathcal{C}_{2} \subset \mathcal{C}_{1}$ and $\mathcal{C}_{3}=\mathcal{C}_{1} \backslash \mathcal{C}_{2}$. If both $\mathcal{C}_{1}$ and $\mathcal{C}_{2}$ are hereditary with respect to induced subgraphs and if $\mathcal{C}_{1}$ corresponds to an $N P$ complete sandwich problem, then $\mathcal{C}_{3}$ also corresponds to an NP-complete sandwich problem. Indeed, we can construct a polynomial-time reduction from GRAPH SANDWICH PROBLEM FOR $\mathcal{C}_{1}$ GRAPHS to GRAPH SANDWICH PROBLEM FOR $\mathcal{C}_{3}$ GRAPHS by using a graph of class $\mathcal{C}_{1}$ that is a forbidden subgraph for class $\mathcal{C}_{2}$. We emphasize that the present paper investigates the case where $\mathcal{C}_{1}$ is not hereditary with respect to induced subgraphs. Indeed, again consider three graph classes, say $\mathcal{C}_{1}, \mathcal{C}_{2}, \mathcal{C}_{3}$, such that $\mathcal{C}_{2} \subset \mathcal{C}_{1}$ and $\mathcal{C}_{3}=\mathcal{C}_{1} \backslash \mathcal{C}_{2}$. If both $\mathcal{C}_{1}$ and $\mathcal{C}_{2}$ correspond to polynomial recognition problems, then $\mathcal{C}_{3}$ does correspond to a polynomial recognition problem as well. For the corresponding sandwich problems, we propose the study of how the complexity of the sandwich problems for $\mathcal{C}_{1}$ and $\mathcal{C}_{2}$ may determine the complexity of the sandwich problem for $\mathcal{C}_{3}$, even if both $\mathcal{C}_{1}$ and $\mathcal{C}_{2}$ correspond to polynomial sandwich problems: given an instance of the sandwich problem for $\mathcal{C}_{1}$, it may admit a sandwich graph that belongs to $\mathcal{C}_{2}$ and another sandwich graph that belongs to $\mathcal{C}_{3}$.

\section{ACKNOWLEDGMENTS}

The work was partially supported by CAPES, CNPq and FAPERJ. We are grateful to the referees for their careful reading and the many suggestions which helped to improve the paper. 


\section{REFERENCES}

[1] L. Alcón, L. Faria, C. M. H. de Figueiredo, M. Gutierrez. Clique graph recognition is NP-complete. In Proc. WG 2006, Lecture Notes in Comput. Sci., vol. 4271, pages 269-277, 2006.

[2] C. Berge and P. Duchet. A generalization of Gilmore's theorem. In Recent Advances in Graph Theory, pages 49-55, Acad. Praha, Prague, 1975.

[3] A. Brandstädt, V.B. Le, J.P. Spinrad. Graph Classes: A survey. SIAM Monographs on Discrete Mathematics and Applications, 1999.

[4] S. Dantas, L. Faria, and C. M. H. de Figueiredo. On decision and optimization $(k, l)$-graph sandwich problems. Discrete Appl. Math., 143:155-165, 2004.

[5] M. C. Dourado, F. Protti, J. L. Szwarcfiter. Computational aspects of the Helly property: a Survey. Journal of the Brazilian Computer Society, 12:7-33, 2006.

[6] F. F. Dragan. Centers of Graphs and the Helly Property (in Russian). Doctoral Thesis, Moldava State University, Chisinău, 1989.

[7] C. M. H. de Figueiredo, S. Klein, and K. Vusković. The graph sandwich problem for 1-join composition is NP-complete. Discrete Appl. Math., 121:73-82, 2002.

[8] M. C. Golumbic, H. Kaplan, and R. Shamir. Graph sandwich problems. J. of Algorithms, 19:449-473, 1995.

[9] M. C. Golumbic and A. Wassermann. Complexity and algorithms for graph and hypergraph sandwich problems. Graphs Combin., 14:223-239, 1998.

[10] M. Gutierrez and J. Meidanis. On the clique operator. In Proc. Latin'98: Theoretical Informatics, Lecture Notes in Comput. Sci., vol. 1380, pages 261272,1998

[11] H. Kaplan and R. Shamir. Bounded degree interval sandwich problems. Algorithmica, 24:96-104, 1999.

[12] T. A. McKee, F. R. McMorris. Topics in Intersection Graph Theory. SIAM Monographs on Discrete Mathematics and Applications, 1999.

[13] E. Prisner. Hereditary clique-Helly graphs. J. Combin. Math. Combin. Comput., 14:216-220, 1993.
[14] E. Prisner. A common generalization of line graphs and clique graphs, J. Graph Theory, 18:301-313, 1994.

[15] E. Prisner. Graph Dynamics. Pitman Research Notes in Mathematics 338, Longman, 1995.

[16] F. S. Roberts and J. H. Spencer. A characterization of clique graphs. J. Combin. Theory B, 10:102-108, 1971.

[17] J. L. Szwarcfiter. Recognizing clique-Helly graphs. Ars Combinatoria, 45:29-32, 1997.

[18] J. L. Szwarcfiter. A survey on clique graphs. In Recent Advances in Algorithms and Combinatorics, pages 109-136, Springer (CMS books in Mathematics), 2003.

[19] W. D. Wallis and G. H. Zhang. On maximal clique irreducible graphs. J. Combin. Math. Combin. Comput., 8:187-193, 1990. 